\title{
The Role of Home Blood Pressure Telemonitoring for Blood Pressure Control
}

\author{
Chan Joo Lee Sungha Park
}

Division of Cardiology, Severance Cardiovascular Hospital and Cardiovascular Research Institute, Yonsei University College of Medicine, Seoul, Republic of Korea

\section{Key Words}

Hypertension · Blood pressure control · Home blood pressure telemonitoring ·

Self-measurement of blood pressure $\cdot$ Remote physician care

\begin{abstract}
Despite improvements in hypertension awareness and treatment, the blood pressure (BP) control rate still remains at around $50 \%$. One of the major determinants of low BP control results from therapeutic inertia of the physician and suboptimal compliance of the patients. Home BP self-measurement and monitoring improves patients' awareness and helps the management of hypertension. Recent technological advances are allowing for accurate measurement and telemonitoring of home $\mathrm{BP}$, with a number of randomized clinical trials demonstrating the efficacy of telemonitoring for BP control. Home BP telemonitoring combined with self-adjustment based on prespecified treatment algorithms has been shown to improve BP control. Additionally, telemonitoring with active intervention by medical professionals has been shown to improve drug compliance and increase the target BP achievement rate. Although nothing can replace the tried and tested doctor-patient relationship in the office, telemonitoring of home BP will be an important tool for treating hypertension in the future.
\end{abstract}

\section{Introduction}

Hypertension is the most prevalent risk factor for cardiovascular disease [1]. Blood pressure (BP) control to below the target BP level is important for improving cardiovascular prognosis [2]. However, despite the improvement of hypertension control rate over the past several decades, the BP control rate still remains low at around 50\% [1, 3]. Important factors 
for the low BP control rate are therapeutic inertia of the attending physician and suboptimal medication compliance of the patient [4]. Home BP self-monitoring may be one way to improve both adherence and BP control. In a systemic review and meta-analysis reported by Agarwal et al. [4], there was a significant improvement in both systolic BP (SBP, $-2.63 \mathrm{~mm}$ $\mathrm{Hg}$; $95 \%$ confidence interval, $\mathrm{CI},-4.24$ to $-1.02 \mathrm{~mm} \mathrm{Hg}$ ) and diastolic BP (DBP, $-1.68 \mathrm{~mm} \mathrm{Hg}$; $95 \% \mathrm{CI}-2.58$ to $-0.79 \mathrm{~mm} \mathrm{Hg}$ ) with home-based BP compared with clinic-based measurements (control group). Home BP monitoring led to significant reduction in antihypertensive medications (relative risk, RR, 2.02; 95\% CI 1.32-3.11) along with less therapeutic inertia (RR for unchanged medication, $0.82 ; 95 \%$ CI 0.68-0.99) [4]. However, self-measurement of home $\mathrm{BP}$ without some forms of active monitoring by medical personnel may not be enough to improve BP control. In the Treatment of Hypertension Based on Home or Office Blood Pressure (THOP) trial, a randomized study that compared BP control rate in 203 subjects whose BP medication was adjusted according to home BP compared to 197 control subjects, medication adjustment according to home BP measurements led to less intensive drug treatment but with less BP control [5]. This demonstrates that home BP telemonitoring, behavioral modification as well as algorithm for medication adjustment are needed for improvement in BP control. In this mini-review, we will discuss recent data regarding the effectiveness of home $\mathrm{BP}$ telemonitoring for BP control.

\section{Telemonitoring of Home BP: Its Effectiveness in Improving BP Control Rate}

The recent development of wireless technologies along with mobile telephone-based transmission systems allows for collection of home BP measurement data into the remote computer server of the health care provider [6]. These recent technological innovations have resulted in telemonitoring that can provide advices regarding adjustments in medications as well as behavioral alterations, both through intervention by medical professionals as well as by self-adjustment based on a prespecified algorithm [7-9]. In contrast to self-monitoring of home BP, telemonitoring of home BP improves BP control. In a meta-analysis by Omboni et al. [10], despite the high level of heterogeneity among the analyzed studies, there was a significant reduction in office BP (SBP, $5.64 \mathrm{~mm} \mathrm{Hg}$; 95\% CI 3.36-7.92; DBP, $2.78 \mathrm{~mm} \mathrm{Hg}$; 95\% CI 1.62-3.93; $n=4,389$ ) and a significant reduction in ambulatory SBP in the home BP telemonitoring group (SBP, $2.28 \mathrm{~mm} \mathrm{Hg;} \mathrm{95 \%} \mathrm{CI} \mathrm{0.24-4.32;} \mathrm{n}=655$ ). The telemonitoring group had a 31\% higher target BP achievement at a cost of significant increase in the use of antihypertensive medications [10]. However, to have a significant BP lowering effect, the telemonitoring needed to be combined with some sort of intervention, be it a self-titration protocol or intervention by a health care professional. In another meta-analysis of 23 randomized trial by Omboni et al. [11], home BP telemonitoring improved office SBP by $4.71 \mathrm{~mm} \mathrm{Hg} \mathrm{(95 \%} \mathrm{CI}$ 3.24-6.18, p < 0.001) and office DBP by $2.45 \mathrm{~mm} \mathrm{Hg}$ (95\% CI 1.57-3.33, p < 0.001), with a larger proportion of patients achieving BP below the target BP (RR, 1.16; 95\% CI 1.04-1.29; $\mathrm{p}<0.001$ ). However, this was associated with significantly increased health care costs (+662.92 EUR per patients; 95\% CI 540.81-785.04) [11].

\section{Effectiveness of Active Intervention by Medical Personnel Based on Home BP Telemonitoring}

The effectiveness of home BP telemonitoring combined with self-management based on a titration algorithm was determined in the Telemonitoring and Self-Management in the Control of Hypertension (TASMINH2) trial, which enrolled 527 participants and randomized 
Lee and Park: The Role of Home Blood Pressure Telemonitoring for Blood Pressure Control

them to self-management of their anti-hypertensive medications $(n=263)$ or control $(n=$ 264). The intervention by the research group based on the telemonitored home BP was limited to checking for the patient's compliance to the safety advice for high or low readings [12]. The results showed that mean SBP at 6 months decreased by $3.7 \mathrm{~mm} \mathrm{Hg}$ (95\% CI 0.8-6.6; $\mathrm{p}=0.013$ ) in the self-management group compared to control. The mean SBP at 12 months also was significantly decreased by $5.4 \mathrm{~mm} \mathrm{Hg}(95 \%$ CI $2.4-8.5 ; \mathrm{p}=0.0004)$ in the selfmanagement group compared to control [12]. Based on these studies, self-management of BP medications based on prespecified algorithm and/or based on recommendations from medical personnel seems to improve BP control. What about the effectiveness of BP reduction when active intervention by medical personnel is done based on home BP telemonitoring? The Hypertension Intervention Nurse Telemedicine Study (HINTS) was a trial evaluating the effectiveness of three telemonitoring-based interventions [13]. All patients were provided a home BP monitor (model UA-767PC; A\&D Medical Digital Blood Pressure, Tokyo, Japan) and a wireless home BP telemonitoring device (model 102; Carematrix Inc., Chicago, Ill., USA). The study subjects were randomized to (1) usual care; (2) nurse administered behavioral management; (3) nurse administered, physician directed medication management, and (4) combined interventions of both medication and behavioral managements [13]. At 12 months, both the behavioral management and physician-directed medication management groups showed significant improvements in BP control compared to the usual care group at $12.8 \%$ (95\% CI 1.6-24.1) and 12.5\% (95\% CI 1.3-23.6), respectively. In addition, the subgroup analyses of those with poor baseline BP control revealed that the combined intervention decreased SBP by $14.8 \mathrm{~mm} \mathrm{Hg}(95 \% \mathrm{CI}-21.8$ to -7.8$)$ at 12 months and $8.0 \mathrm{~mm} \mathrm{Hg}(95 \% \mathrm{CI}$ -15.5 to -0.5 ) at 18 months, compared to usual care [13]. Similarly, in a randomized study of 401 subjects with uncontrolled hypertension aged 29-95 years, telemonitoring of home BP by the attending nurses and physicians with automated patient decision support was compared to control for 6 months. The clinicians were allowed to contact the participants if needed to arrange changes in the medications. The results show that the intervention group had a significant reduction of both daytime ambulatory SBP (4.3 mm Hg; 95\% CI 2.0-6.5, p = $0.0002)$ and daytime ambulatory DBP $(2.3 \mathrm{~mm} \mathrm{Hg} ; 95 \% \mathrm{CI} 0.9-3.6 ; \mathrm{p}=0.001)$ [8].

In a study by Green et al. [14], 778 participants with uncontrolled hypertension aged 25-75 years were randomized to either usual care, home BP monitoring with patient website training or home BP monitoring, and secure patient website training with pharmacist care management. The results showed that web-based pharmacist care was associated with a significantly increased percentage of patients with controlled BP (56\%; 95\% CI 49-62) compared to both usual care (31\%; 95\% CI 25-37; p < 0.001) and home BP monitoring with web training only (36\%; 95\% CI 30-42; $<<0.001)$. In patients who had baseline SBP of higher than $160 \mathrm{~mm} \mathrm{Hg}$, web-based pharmacist care was associated with a significant net reduction of both SBP ( $-13.2 \mathrm{~mm} \mathrm{Hg}$; 95\% CI -19.2 to $-7.1 ; \mathrm{p}<0.001)$ and DBP $(-4.6 \mathrm{~mm} \mathrm{Hg}$; $95 \% \mathrm{CI}$ -8.0 to $-1.2 ; \mathrm{p}<0.001$ ) [14]. Similarly, a cluster randomized trial of 450 adults with uncontrolled hypertension were randomized to either usual care $(n=222)$ or telemonitoring intervention and pharmacist management $(n=228)$ [15]. The results showed that the proportion of subjects with composite BP control at both 6 and 12 months was significantly greater in the intervention arm (57.2 vs 30.0\%; $p=0.001$ ) (table 1; reproduced from Margolis et al. [15], with permission from the American Medical Association). In addition, the BP control rate at 6,12 , and 18 months was significantly higher in the intervention arm as well (table 1). Compared to the usual care group, there was a significant decrease in SBP at 6 months $(-10.7$ mm Hg; 95\% CI -14.3 to -7.3; p < 0.001), 12 months (-9.7 mm Hg; 95\% CI -13.4 to $-6.0 ; \mathrm{p}<$ $0.001)$, and 6 months ( $-6.6 \mathrm{~mm} \mathrm{Hg} ; 95 \% \mathrm{CI}-10.7$ to $-2.5 ; \mathrm{p}=0.004)$. The effectiveness of the intervention arm 6 month after the end of the trial (18th month of the trial) again demonstrates the sustained effect of telemedicine after the end of the active monitoring [15]. 
Table 1. Composite and BP control by telemonitoring intervention or usual care (reproduced from Margolis et al. [15], with permission from the American Medical Association)

\begin{tabular}{|c|c|c|c|c|c|c|}
\hline & \multicolumn{2}{|c|}{$\begin{array}{l}\text { Telemonitoring } \\
\text { intervention }\end{array}$} & \multicolumn{2}{|c|}{ Usual care } & \multirow{2}{*}{$\begin{array}{l}\text { Differential } \\
\text { change from } \\
\text { baseline, } \\
\%(95 \% \mathrm{CI})\end{array}$} & \multirow[t]{2}{*}{$\mathrm{p}$ value $^{\mathrm{a}}$} \\
\hline & $\begin{array}{l}\text { Pa- } \\
\text { tients, } \mathrm{n}\end{array}$ & $\%(95 \% \mathrm{CI})$ & $\begin{array}{l}\mathrm{Pa}- \\
\text { tients, } \mathrm{n}\end{array}$ & $\%(95 \% \mathrm{CI})$ & & \\
\hline \multicolumn{7}{|l|}{ Composite BP control } \\
\hline At 6 and 12 months & 113 & $57.2(44.8-68.7)$ & 58 & $30.0(23.2-37.8)$ & $27.2(13.4-40.0)$ & 0.001 \\
\hline At 6,12 , and 18 months & 96 & $50.9(36.9-64.8)$ & 42 & $21.3(14.4-30.4)$ & $29.6(13.1-46.0)$ & 0.002 \\
\hline \multicolumn{7}{|l|}{ BP control } \\
\hline At 6 months & 148 & $71.8(65.6-77.3)$ & 89 & $45.2(39.2-51.3)$ & $26.6(19.2-33.1)$ & $<0.001$ \\
\hline At 12 months & 141 & $71.2(62.0-78.9)$ & 102 & $52.8(45.4-60.2)$ & $18.4(7.9-27.0)$ & 0.005 \\
\hline At 18 months & 135 & $71.8(65.0-77.8)$ & 104 & $57.1(51.5-62.6)$ & $14.7(7.0-21.4)$ & 0.003 \\
\hline
\end{tabular}

a Study group difference for composite BP control and at each individual time point.

What about the effectiveness of telemonitoring with remote, web-based management by the attending physician? Recently, we performed a study to determine the effectiveness of remote patient monitoring and physician care for treatment of hypertension. A total of 374 hypertensive patients aged 20 years and over were randomized into three groups: (1) control, patients received usual clinical care with home BP monitoring; (2) patients were remotely monitored and received office follow-up, and (3) patients received remote monitoring with out-of-office physician care using the remote monitoring device, respectively [9]. The results did not show any significant difference between the three groups for the primary endpoint, adjusted mean sitting SBP (group 1, $-8.9 \pm 15.5 \mathrm{~mm} \mathrm{Hg}$; group 2, $-11.3 \pm 15.9 \mathrm{~mm} \mathrm{Hg}$; group $3,-11.6 \pm 19.8 \mathrm{~mm} \mathrm{Hg}$; $\mathrm{p}=$ n.s.). However, achieving the target BP at the 24 th week of followup was significantly higher in group 2 compared to group 1 . In addition, subgroup analysis of subjects aged over 55 years showed a significant decrease in adjusted mean sitting SBP in groups 2 and 3 compared to the control. The study demonstrated that remote monitoring combined with remote physician care was efficacious and safe for reducing BP in patients with hypertension as usual care [9]. Table 2 summarized the study population, outcome, and major findings from all studies mentioned in this mini-review (table 2). From all evidence mentioned previously, it seems that home BP telemonitoring improves BP control when compared to usual care.

Nonetheless, whether home BP telemonitoring improves the BP control rate compared to home BP monitoring alone is not clear. In a meta-analysis by Uhlig et al. [16], 52 prospective studies comparing self-measured BP (SMBP) with or without additional supportive care with usual care were analyzed. The study found that SMBP monitoring with additional supportive care lowers SBP by -3.4 to $-8.9 \mathrm{~mm} \mathrm{Hg}$ and DBP by -1.9 to $-4.4 \mathrm{~mm} \mathrm{Hg}$ at up to 12 months. However, the same study did not find any evidence that SMBP plus additional supportive care lowers BP better than SMBP alone [16]. When considering the evidence at hand, more data is needed to show the benefit of home BP telemonitoring with or without additional care in improving both BP control and prognosis compared to usual care. Until that time, home BP self-monitoring should still be the out-of-office BP monitoring of choice in the management of hypertension [17]. Though, the evidence suggests that home BP telemonitoring may be useful in selected circumstances such as in sparsely populated areas where medical care access is not readily available and in cases of natural or man-made disasters. For example, during the aftermath of the devastating tsunami in Tohoku, Japan, investigators have 


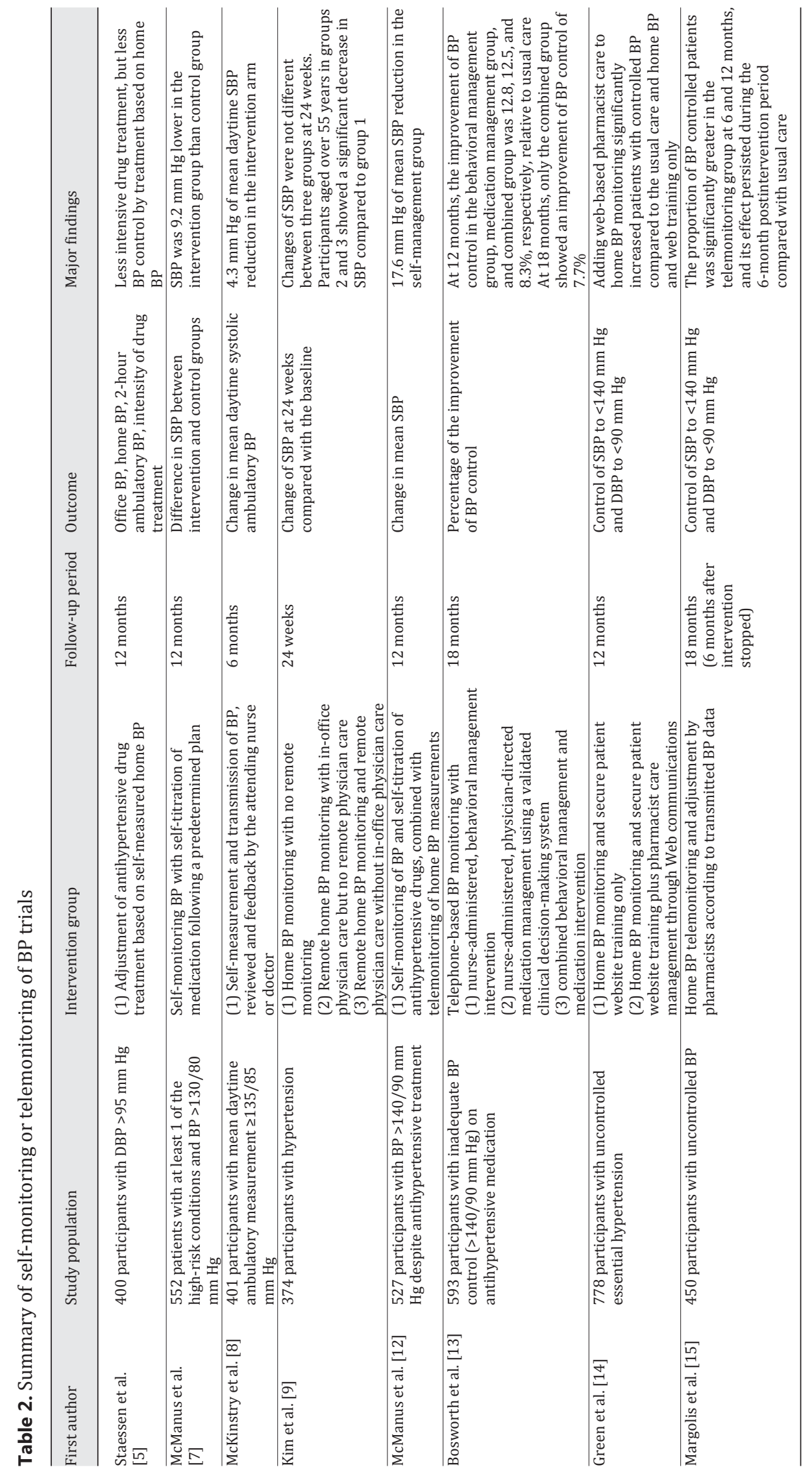


Lee and Park: The Role of Home Blood Pressure Telemonitoring for Blood Pressure Control

developed a web-based Disaster Cardiovascular Prevention (DCAP) network to monitor cardiovascular risk factors in the survivors of the tsunami and to promote preventive behaviors [18]. In the future, with ever improving technological advances, application of telemonitoring for treatment of chronic diseases, such as hypertension, will only increase. We as clinicians should be ready for the upcoming changes.

\section{Conclusion}

The treatment of hypertension based on measurement of office BP once every few months may be a thing of the past. Home BP monitoring is a cost-effective way to assess for white coat hypertension, masked hypertension, and 24-hour BP control while increasing patient awareness and treatment adherence. Therefore, home BP should be the ideal out-of-office BP measurement for routine management of hypertension patients [19]. At present, when considering the evidence and the cost-effectiveness, co-intervention, including teletransmission or health care professional co-management, should be limited to selected cases. However, technological advances are allowing for accurate measurement and telemonitoring of day-to-day home BP. Also, active intervention of medical personnel through the use of BP telemonitoring is helpful for improving drug compliance and achieving target BP. Although nothing can replace the tried and tested doctor-patient relationship in the office, telemonitoring of home BP will be an important tool for treating hypertension in the future.

\section{Acknowledgement}

This work was supported by a grant from the Korean Health Technology R \& D Project, Ministry of Health and Welfare, Republic of Korea (HI13C0715).

\section{Disclosure Statement}

The authors have no financial conflict of interests that are related to this subject.

\section{References}

1 Egan BM, Zhao Y, Axon RN: US trends in prevalence, awareness, treatment, and control of hypertension, 19882008. JAMA 2010;303:2043-2050.

-2 Mancia G, Fagard R, Narkiewicz K, Redon J, Zanchetti A, Bohm M, et al: 2013 ESH/ESC Guidelines for the management of arterial hypertension: the Task Force for the management of arterial hypertension of the European Society of Hypertension (ESH) and of the European Society of Cardiology (ESC). J Hypertens 2013; 31:1281-1357.

-3 Joffres M, Falaschetti E, Gillespie C, Robitaille C, Loustalot F, Poulter N, et al: Hypertension prevalence, awareness, treatment and control in national surveys from England, the USA and Canada, and correlation with stroke and ischaemic heart disease mortality: a cross-sectional study. BMJ Open 2013;3:e003423.

4 Agarwal R, Bills JE, Hecht TJ, Light RP: Role of home blood pressure monitoring in overcoming therapeutic inertia and improving hypertension control: a systematic review and meta-analysis. Hypertension 2011;57: 29-38.

5 Staessen JA, Den Hond E, Celis H, Fagard R, Keary L, Vandenhoven G, et al: Antihypertensive treatment based on blood pressure measurement at home or in the physician's office: a randomized controlled trial. JAMA 2004;291:955-964.

-6 Omboni S, Ferrari R: The role of telemedicine in hypertension management: focus on blood pressure telemonitoring. Curr Hypertens Rep 2015;17:535.

7 McManus RJ, Mant J, Haque MS, Bray EP, Bryan S, Greenfield SM, et al: Effect of self-monitoring and medication self-titration on systolic blood pressure in hypertensive patients at high risk of cardiovascular disease: the TASMIN-SR randomized clinical trial. JAMA 2014;312:799-808. 
Lee and Park: The Role of Home Blood Pressure Telemonitoring for Blood Pressure Control

8 McKinstry B, Hanley J, Wild S, Pagliari C, Paterson M, Lewis S, et al: Telemonitoring based service redesign for the management of uncontrolled hypertension: multicentre randomised controlled trial. BMJ 2013;346:f3030.

-9 Kim YN, Shin DG, Park S, Lee CH: Randomized clinical trial to assess the effectiveness of remote patient monitoring and physician care in reducing office blood pressure. Hypertens Res 2015;38:491-497.

$\checkmark 10$ Omboni S, Guarda A: Impact of home blood pressure telemonitoring and blood pressure control: a metaanalysis of randomized controlled studies. Am J Hypertens 2011;24:989-998.

11 Omboni S, Gazzola T, Carabelli G, Parati G: Clinical usefulness and cost effectiveness of home blood pressure telemonitoring: meta-analysis of randomized controlled studies. J Hypertens 2013;31:455-467; discussion 67-68.

12 McManus RJ, Mant J, Bray EP, Holder R, Jones MI, Greenfield S, et al: Telemonitoring and self-management in the control of hypertension (TASMINH2): a randomised controlled trial. Lancet 2010;376:163-172.

-13 Bosworth HB, Powers BJ, Olsen MK, McCant F, Grubber J, Smith V, et al: Home blood pressure management and improved blood pressure control: results from a randomized controlled trial. Arch Intern Med 2011;171: 1173-1180.

14 Green BB, Cook AJ, Ralston JD, Fishman PA, Catz SL, Carlson J, et al: Effectiveness of home blood pressure monitoring, web communication, and pharmacist care on hypertension control: a randomized controlled trial. JAMA 2008;299:2857-2867.

15 Margolis KL, Asche SE, Bergdall AR, Dehmer SP, Groen SE, Kadrmas HM, et al: Effect of home blood pressure telemonitoring and pharmacist management on blood pressure control: a cluster randomized clinical trial. JAMA 2013;310:46-56.

16 Uhlig K, Patel K, Ip S, Kitsios GD, Balk EM: Self-measured blood pressure monitoring in the management of hypertension: a systematic review and meta-analysis. Ann Intern Med 2013;159:185-194.

17 Imai Y, Obara T, Asamaya K, Ohkubo T: The reason why home blood pressure measurements are preferred over clinic or ambulatory blood pressure in Japan. Hypertens Res 2013;36:661-672.

18 Kario K, Nishizawa M, Hoshide S, Shimpo M, Ishibashi Y, Kunii O, et al: Development of a disaster cardiovascular prevention network. Lancet 2011;378:1125-1127.

$\checkmark 19$ Tientcheu D, Ayers C, Das SR, McGuire DK, de Lemos JA, Khera A, et al: Target organ complications and cardiovascular events associated with masked hypertension and white-coat hypertension: analysis from the Dallas Heart Study. J Am Coll Cardiol 2015;66:2159-2169. 